\title{
Liquid State Anomalies and the Relationship to the Crystalline Phase Diagram.
}

\author{
Domagoj Fijan and Mark Wilson ${ }^{1}$ \\ ${ }^{1}$ Department of Chemistry, Physical and Theoretical Chemistry Laboratory, \\ University of Oxford, South Parks Road, Oxford OX1 3QZ, UK
}

\begin{abstract}
A relationship between the observation of a density anomaly and the underlying crystalline phase diagram is demonstrated. The crystal phase diagram and temperature of maximum density (TMD) lines are calculated over a range of parameter space using a Stillinger-Weber potential. Relationships between the loci of density maxima in the $P T$ plane for the liquid state and the underlying crystalline phase diagram are investigated. Two key potential parameters are systematically varied in order to control the balance between the model two- and three-body interaction terms, and the relative effects of varying the potential parameters analysed. The respective TMD lines diverge at extreme values with one set of lines showing a re-entrant behaviour. For each parameter set the TMD lines are extrapolated to $T=0 \mathrm{~K}$. The corresponding pressures are related to the crystalline phase diagram and are found to lie on or near specific crystal/crystal coexistence lines for a wide range of potential parameters. The density anomaly is observed to vanish corresponding to regions in the crystal phase diagram which lack crystal/crystal coexistence lines potentially offering a new interpretation for the emergence of anomalous behaviour.
\end{abstract}

The origin of anomalous behaviour in liquids is a long standing mystery in field of condensed matter and, significantly, in the water community. Although exhaustive thermodynamical studies have been performed ${ }^{1}$ and significant progress made, no unified theory as to the origin of anomalous behaviour exists. Recently the focus has shifted towards studying the relationship between anomalies and liquid structure ${ }^{2-6}$. The two-state model has, for example, proved to be successful in tackling many of the intricacies of this problem ${ }^{7}$ leading to identification of specific local environments that relate to the observed anomalous behaviour. To date there has been relatively little work which considers any relationship between the coexistence lines on the crystalline phase diagram and lines that define the thermodynamic anomalies ${ }^{8,9}$. Previous work relies on the observation that the systems which show anomalies have more than one clearly related and thermodynamically accessible crystal structure ${ }^{8,9}$.

Crystalline (highly ordered) and amorphous or liquid (disordered) structures may be reasonably expected to show significant similarities. However, connections between the evolution of liquid structure with state point (which is generally continuous) and any underlying crystalline phase transformations (which are generally discontinuous) are often unclear. Clear links should be possible given that the interactions in the different states are likely to be similar (in the absence of, for example, traversing a metal-insulator transition). Extensions of the phase diagram into the thermodynamically metastable regimes (for example, on supercooling) may uncover a richness of phase behaviour, showing distinct amorphous regions which differ in density and show welldefined phase boundaries ${ }^{10}$. A prevalent characteristic is a balance between forming relatively low density ("open") structures at low pressures and significantly higher density ("close-packed") structures at higher pressures. This balance is mirrored in the crystalline phase diagram and in the metastable supercooled amorphous regimes. Furthermore, systems of this type often display pressure-induced amorphisation in which an amorphous structure is generated directly from a crystal on application of pressure ${ }^{11-13}$. Network systems (e.g. Si, Ge, $\mathrm{H}_{2} \mathrm{O}, \mathrm{SiO}_{2}, \mathrm{GeO}_{2}$ and $\mathrm{BeF}_{2}$ ) also often display anomalous properties which may extend into both the structure and dynamics. Of these, perhaps the most well-known anomaly is in the density of $\mathrm{H}_{2} \mathrm{O}$ (see, for example, ref. ${ }^{13}$ and references therein). Constructing a locus of the turning points (usually in the $P T$ or $\rho T$ planes) generates a temperature of maximum density (TMD) line. Further anomalies are observed in, for example, the heat capacity, isothermal compressibilities and diffusivities ${ }^{13-31}$. The origins and relationships between these anomalies appear complex, potentially arising from a subtle disruption of the tetrahedral network and have been studied using accurate models for $\mathrm{SiO}_{2}{ }^{18,19,27,30}, \mathrm{BeF}_{2}{ }^{28-30}, \mathrm{GeO}_{2}{ }^{30}$ and $\mathrm{H}_{2} \mathrm{O}^{3,6,13,15-17,30,32}$ and their evolution traced using the Stillinger-Weber potential ${ }^{33,34}$ and using ramp ram, $^{27-37}$ or core-softened $^{38}$ potentials. Potential models, in which the energy of interaction is deconvoluted into a series of $n$-body $(n \geq 2)$ interactions are attractive both for their relative simplicity (computational efficiency) and for the ability to control fundamental interactions in a well-defined manner. For example, many of the issues outlined above may be considered insightfully using potential models in which the two- and three-body interactions are determined explicitly and their relative magnitudes varied.

In this work we present systematic numerical evidence for the conditions under which the density anomaly emerges in systems modelled with a Stillinger-Weber (SW) potential ${ }^{39}$. The purpose of this work is to outline that the origin of anomalous behaviour can be traced back to elementary properties of the underlying crystalline phase diagram rather than relying on a more standard "hunt" for the source of this behaviour ( for example, searching for the liquid-liquid critical point). We systematically explore the liquid and crystalline phase space and the model parameter space by tuning two dif- 
ferent physically intuitive parameters in the SW model. The SW model is a widely used potential that can effectively model a range of monatomic systems which are based on local tetrahedral geometries, including silicon ${ }^{39}$, phosphorus $^{40,41}$, germanium ${ }^{42}$, carbon $^{43}$, water $^{44}$ and even a range of multicomponent systems ${ }^{41,45,46}$. Here we extend the studies to non-tetrahedral local environments, offering the possibility of exploring a range of additional systems. We focus on the TMD lines as a signature of significant structural change in the disordered environment and consider their relationship to the underlying crystalline phase diagram by extrapolating to $T=0 \mathrm{~K}$ in order to uncover links with the crystalline phase diagrams as well as relating to changes in the local structure. A Stillinger-Weber potential ${ }^{39}$ is employed throughout as an example of a well-studied model known to show key anomalous properties and in which the relative strengths of the 2- and 3-body terms is easily controlled $33,34,42,47-50$. The two key parameters varied (labelled $\lambda$ and $\theta_{0}$ ) control the strength of the three-body term and the preferred local bond angle. The effect of varying $\lambda$ (with $\theta_{0}$ set at the ideal tetrahedral angle, $\theta_{T_{d}}$ ) has been considered previously ${ }^{33,34,42,47-49}$ whilst the effect of varying $\theta_{0}$ has been less well studied ${ }^{50}$. A study using a somewhat different model (a Kern-Frenkel model ${ }^{51}$ ) which focused on the liquid-liquid critical point progression as a function of the angle of interaction has been reported $^{52}$.

Figure 1 shows the crystal phase diagrams obtained at $T=0 \mathrm{~K}$ both as a function of $\lambda$ and $\theta_{0}$ with all other parameters fixed at the values used to model silicon ${ }^{39}$. The phase diagrams are constructed by determining the energy/volume curves for each potential crystal structure. For structures which show either orthorhombic or tetragonal symmetry different ratios of unit cell lengths are considered and the lowest free energy at a given volume identified. The system pressure is calculated from the derivative of the energy which allows the free energy to be evaluated and coexistence lines to be constructed. A useful feature of this approach is that the results are exact in the limit that all the possible crystal structures can be assessed. Consider first the ideal tetrahedral potential model $\left(\theta_{0}=\theta_{T_{d}}\right)$ as a function of $\lambda$ (figure 1(a)). At high $\lambda$ the diamond crystal structure becomes stable, reflecting the relatively large energetic penalty imposed on local configurations which deviate from the ideal tetrahedron. For $\lambda<27$ a richer phase diagram emerges in which the thermodynamically-stable crystal structures reflect a balance between the close-packed and more open structures favoured by the two- and three-body interactions respectively. At (small) negative pressures the low density clathrate (SII and SIII) structures are stable. At positive pressures the BC8, SC16 and o- $\mathrm{X}$ crystal structures become stable. These all have distorted tetrahedral nearest-neighbour topologies with a relatively short next-nearest (fifth) neighbour length-scale ${ }^{53-57}$. The o-X crystal structure has been recently characterised ${ }^{57}$ (having been first observed theoretically ${ }^{55}$ ) and can be con-

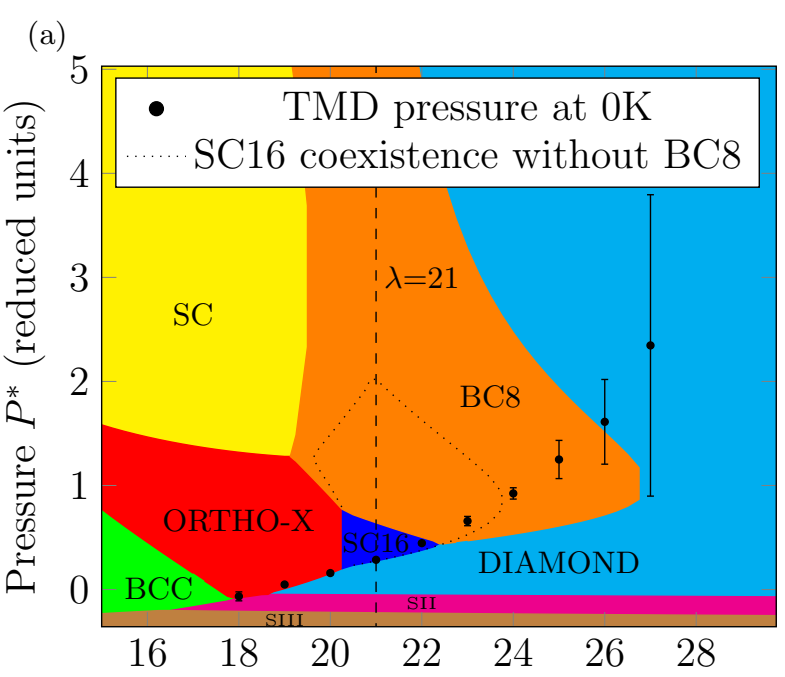

Strength of tetrahedral interactions $\lambda$

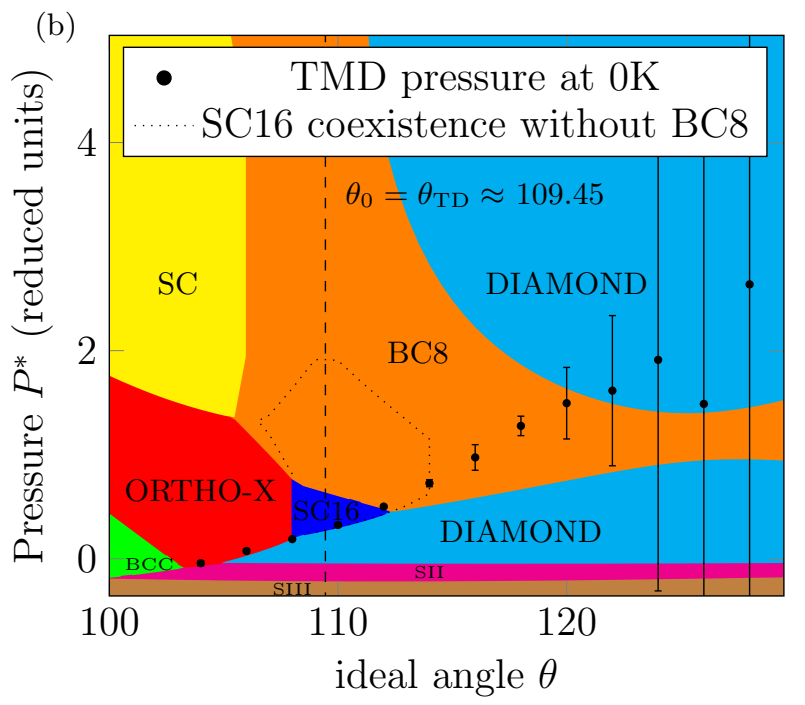

FIG. 1. Crystal phase diagrams determined at $T=0 \mathrm{~K}$ for the Stillinger-Weber potential and shown as a function of the reduced pressure, $p^{*}$, as a function of (a) the magnitude of the three-body interaction energy (as controlled by the parameter $\lambda$ ), and (b) the favoured angle formed by atoms in the local coordination polyhedra (as controlled by the parameter $\theta_{0}$ ). The dotted lines highlight the regions in which the SC16 crystal structure is thermodynamically stable over the diamond structure but metastable with respect to the BC8 structure. The dashed lines highlight the $\theta_{0}=\theta_{T_{d}}$ and $\lambda=21$ lines respectively and which represent the line of intersection of the two phase diagrams. In both cases the solid circles highlight the extrapolation of the TMD lines (shown in figure 2) to $T=0 \mathrm{~K}$ as described in the text.

sidered as a distorted $\beta$-Sn crystal structure. The SC16 and $\mathrm{BC} 8$ crystal structures are closely related and hence occupy similar regions of the phase diagrams. The figures also highlight the regions for which the SC16 structure is thermodynamically stable over the diamond structure but metastable with respect to $\mathrm{BC} 8$. At low $\lambda$ structures with nearest-neighbour coordination numbers 
(a)
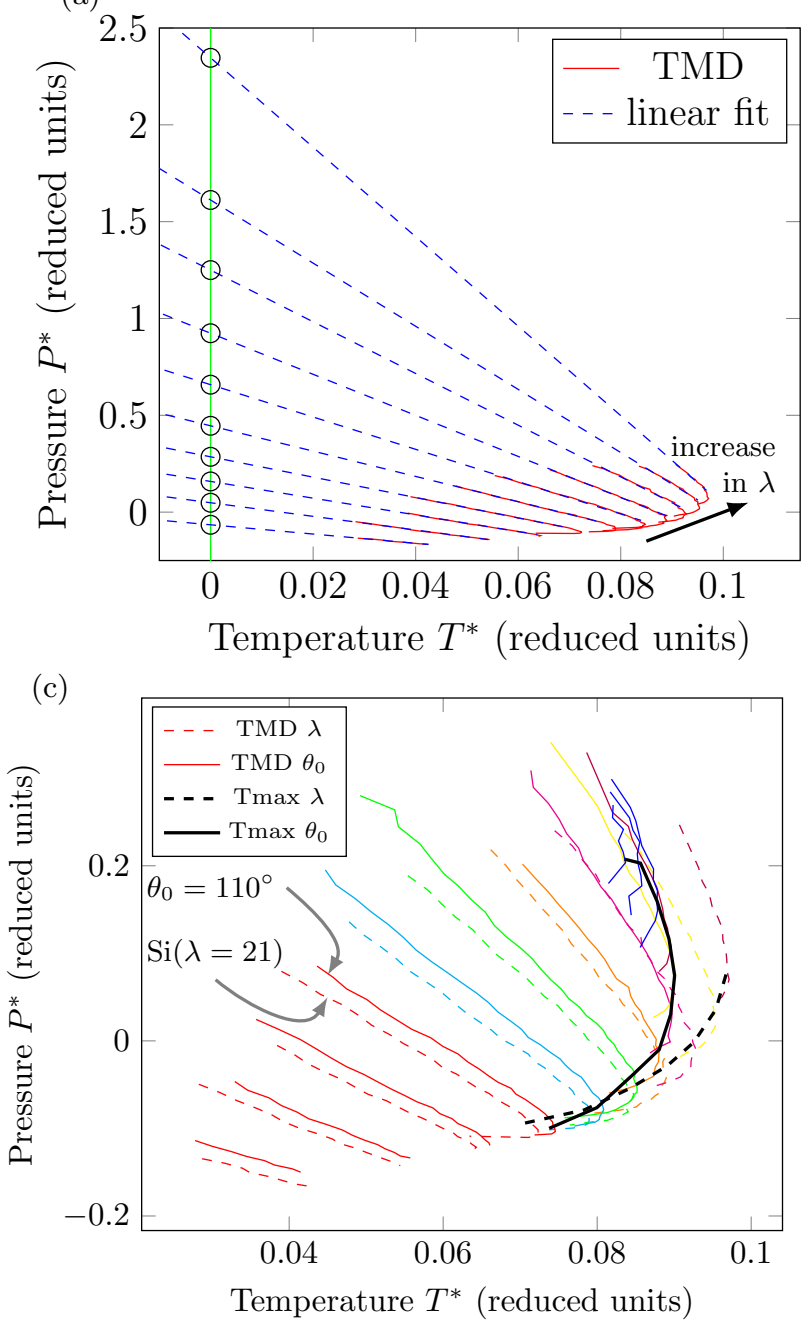

(b)

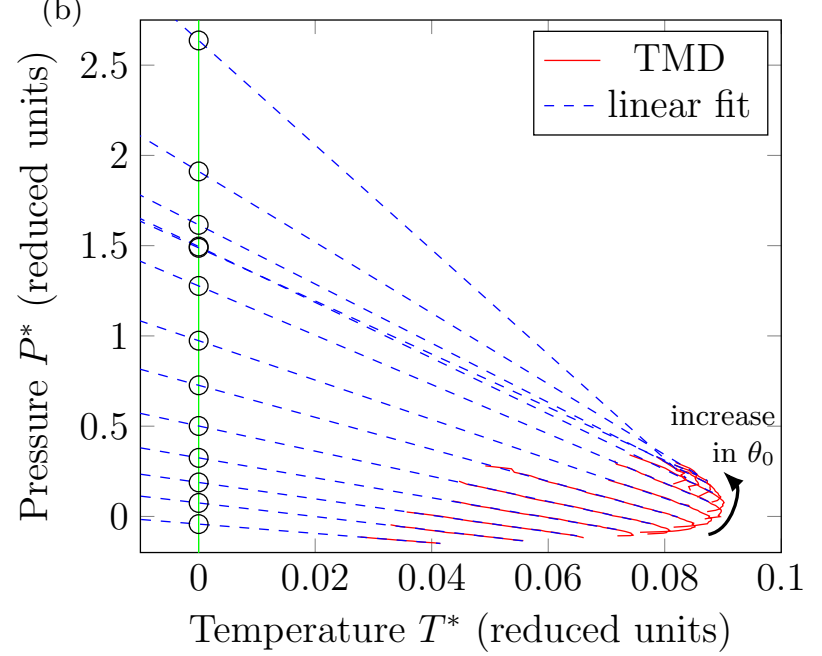

(d)

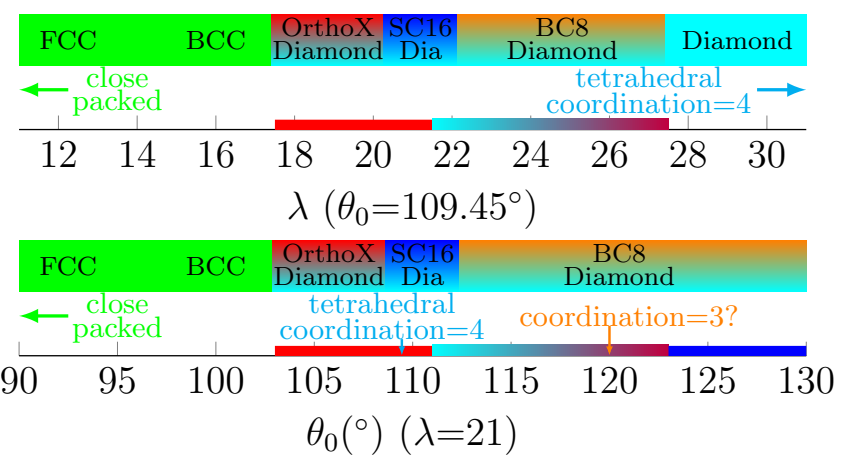

FIG. 2. Temperature of maximum density (TMD) lines determined using a Stillinger-Weber potential and varying (a) $\lambda$ (fixing $\theta_{0}=\theta_{T_{d}}$ ) and (b) $\theta_{0}$ (fixed $\lambda=21$ ) shown as a function of reduced pressure, $P^{*}$, and temperature, $T^{*}$. In panel (a) successive TMD lines are shown for $\lambda=18-27$ (in unit increments) as highlighted, whilst in panel (b) successive lines are shown for $\theta_{0}=104-128^{\circ}$ (in increments of $2^{\circ}$ ). In both panels (a) and (b) the dashed lines show fits to the linear sections of the TMD lines which are used to extrapolate to $T=0 \mathrm{~K}$. (c) Comparison of the progression of the TMD lines for different values of $\lambda$ (dashed) and $\theta_{0}$ (solid). The TMD lines in red show the same trend as a function of either $\lambda$ or $\theta_{0}$, in which increasing the respective parameters shifts the TMD lines to higher pressures and temperatures. The multi-coloured TMD lines highlight the onset of divergent behaviour with increasing $\lambda$ or $\theta_{0}$. The solid blue TMD lines show a progression with increasing $\theta_{0}$ for which no $\lambda$ counterpart has been identified. For these the temperature dependence of the TMD lines appears inverted in that increasing $\theta_{0}$ shifts the lines to lower temperatures and higher pressures. The thick solid lines show the respective envelopes of the maximum temperatures of each TMD line and so highlight the differing evolution of these lines with increasing $\lambda$ and $\theta_{0}$. (d) Summary of the key density anomaly behaviour observed for varying $\lambda$ (upper panel) and $\theta_{0}$ (lower panel). The lower text indicates the local structures observed in the amorphous phase and the upper text the stable crystal phase at $T=0 \mathrm{~K}$ and at the TMD pressure. The red lines highlight the range over which the TMD progressions are consistent in $\lambda$ and $\theta_{0}$. The coloured graduation denotes the onset of divergent behaviour, whilst the blue lines denote the region for which the density anomaly vanishes for $\lambda$ but is retained for $\theta_{0}$. The colours follow the colouring scheme from (c). For the crystal phases the single coloured regions identify single crystal phases whilst the graduated colours denote regions where coexistence between two crystal phases is important. The colours follow the scheme of figure 1 .

greater than four become stable (BCC at low pressure, simple cubic at high pressure) reflecting the increased dominance of the two-body interactions. Figure 1(b) shows the phase diagram at fixed $\lambda(\lambda=21)$ as a function of the local geometry parameter, $\theta_{0}$. Figures $1(\mathrm{a})$ and (b) intersect at $\lambda=21, \theta_{0}=\theta_{T_{d}}$ (as highlighted by dashed vertical lines) and appear to map onto each other at low $\Delta \theta\left(=\theta_{0}-\theta_{T_{d}}\right)$ of $\sim 10^{\circ}$. At first impression this mapping may appear obvious as, in a Stillinger-Weber potential, the total energy is expressed as a sum of ex- 
plicit two- and three-body terms and the magnitude of the latter is controlled both by $\lambda$ and $\theta_{0}$. However, the dependence of the energy on the deviation from $\theta_{0}$ is approximately quadratic (compared to the linear dependence on $\lambda$ ). However, although the dependence of the 3 -body energy term is even about $\theta_{0}$, reducing $\theta_{0}$ will tend to favour the 2-body energy term (as the system can adopt a relatively high coordination number). As a result, lowering $\theta_{0}$ is equivalent in some sense to lowering $\lambda$. At high $\Delta \theta\left(>10^{\circ}\right)$ clear differences in the phase diagrams emerge, for example in the greater range of the stability field for the BC8 structure.

Figure 2 shows the loci of the temperatures at which the system density reaches a maximum, shown in the $P T$ plane for (a) varying $\lambda\left(\theta_{0}=\theta_{T_{d}}\right)$ and (b) varying $\theta_{0}$ $(\lambda=21)$. A point is generated by performing simulations at fixed volumes, varying the temperature, and locating the pressure and temperature at which $(\partial P / \partial T)_{V}=0$. At fixed $\theta_{0}$ (figure $2(\mathrm{a})$ ) TMD lines are observed for $18 \leq \lambda \leq 27$ and shift to higher pressure and temperature as the strength of the three-body interaction increases (reflecting the behaviour of the underlying liquid/gas spinodals ${ }^{48}$ ). For fixed $\lambda$ (figure 2(b)) a slightly different behaviour emerges with TMD lines observed for $104^{\circ} \leq \theta_{0} \leq 128^{\circ}$. For $104^{\circ} \leq \theta_{0} \leq 120^{\circ}$ the TMD lines show analogous behaviour to those for increasing $\lambda$, with successive curves shifting to higher temperature and pressure. For $\theta_{0} \geq 120^{\circ}$ the TMD curves retrace to lower temperature whilst increasing in pressure. To highlight the differences associated with changing both $\lambda$ and $\theta_{0}$ figure $2(\mathrm{c})$ shows the respective TMD lines combined. The low $\lambda$ and $\theta_{0}$ curves effectively map onto each other. However, as both parameters increase the TMD lines diverge with those associated with changes in $\theta_{0}$ showing a re-entrant behaviour, shifting to lower temperature as the pressure increases. Reducing $\lambda$ favours more close-packed structures, which will also be the case for reducing $\theta_{0}$ below $\theta_{T_{d}}$. Increasing $\lambda$ further stabilises the four-coordinate (tetrahedral) sites. However, increasing $\theta_{0}$ "pushes" the favoured structures away from both the close-packed and tetrahedral environments. These observations are summarised in figure $2(\mathrm{~d})$.

The TMDs shown in figure 2 are extrapolated to $T=0 \mathrm{~K}$ by identifying the near-linear regimes and fitting a line using a least-squares procedure. Ideally, we would study the TMD-crystal coexistence relations at finite temperatures. However, assessing a vast range of possible crystal phases at finite temperatures for a wide range of $\lambda$ and $\theta_{0}$ is potentially highly complex. For example, avoiding thermally-driven phase transitions between competing crystal structures (and so obtaining metastable extensions to crystal/liquid coexistence curves) may be problematic. The solution proposed here is to consider the TMD extrapolation to $T=0 \mathrm{~K}$ at which we can determine the phase diagram exactly for a wide range of possible crystal structures. The extrapolation of the TMD to low temperature is not without concerns however. The limit of the TMD locus can terminate in three ways. In the first the line may collide with the $T=0 \mathrm{~K}$ line (the singularity free scenario ${ }^{16}$ ). The gradient of the TMD locus in the PT projection should start to increase as the temperature is lowered. In the second case the TMD may merge with the temperature of minimum density anomaly locus ${ }^{58}$. By construction this must occur at zero gradient in the $P T$ projection which means that the TMD has to increase in gradient. In the third scenario the TMD locus may collide with the spinodal line eminating from the liquid-liquid critical point ${ }^{15}$ or the second liquid-gas critical point ${ }^{13}$. In this scenario an increase in gradient for the TMD locus is also observed. All three possibilities predict an increase in gradient for the TMD locus. However, as we do not know the exact functional form of the TMD line we opt to perform a linear extrapolation in order to obtain an upper pressure limit. Figure 1 shows these extrapolations projected onto the phase diagrams in terms of $\lambda$ (figure $1(\mathrm{a})$ ) or $\theta_{0}$ (figure 1(b)). TMD lines are not observed for $\lambda<18$ and $\theta_{0}<104^{\circ}$ respectively, corresponding to the onset of the BCC crystal stability fields. Furthermore, TMD lines are not observed for $\lambda>27$, in excess of the BC8 crystal stability field (figure 1(a)). TMD lines are observed for high $\theta_{0}$ in line with the increased BC8 crystal stability field (figure 1(b)). In both cases the extrapolated pressures lie close to the respective diamond/o-X and diamond/SC16 coexistence curves for low and intermediate $\left\{\lambda, \theta_{0}\right\}$ respectively. For $\lambda \gtrsim 22$ and $\theta_{0} \gtrsim 112^{\circ}$ the extrapolated TMD points approximately follow the SC16/diamond coexistence curves extended into the regions for which $\mathrm{SC16}$ is metastable with respect to the BC8. For $\lambda \gtrsim 24$ or $\theta_{0} \gtrsim 114^{\circ}$ these points no longer follow a clear coexistence curve but traverse the BC8 stability field. TMD lines are only observed across parameter space for which a non-diamond crystal structure shows a stability field. At high $\lambda(\lambda \gtrsim 27)$ the diamond crystal structure becomes favoured across the whole (positive) pressure range studied. At high $\theta_{0}$ the relative destabilisation of both the close-packed and tetrahedral local coordination environments results in a $\mathrm{BC} 8$ crystal structure thermodynamic stability field which traverses the whole parameter range studied and, as a result, TMD lines are observed across this range.

A common probe employed to evaluate evolution in structure is the radial distribution function (RDF). Figure 3 shows the RDFs and running coordination numbers $\left(n\left(r_{c}\right)=4 \pi \rho \int_{0}^{r_{c}} r^{2} g(r) d r\right)$ for four values of $\theta_{0}$ (at fixed $\lambda=21$ ). For each value of $\theta_{0}$ five functions are shown which cover a range of $\Delta T= \pm 200 \mathrm{~K}$ (equivalent to \pm 0.008 in reduced units) around the temperatures of maximum densities. At each value of $\theta_{0}$ the functions show an isobestic point (a separation at which the coordination number curves approximately intersect $)^{4,59}$. The presence of these points indicates that, at fixed density, there appears an 'optimum' number of atoms within a given cutoff which is approximately temperature-independent. Models which show extensive TMD lines have isobestic coordination numbers in the 
(a)

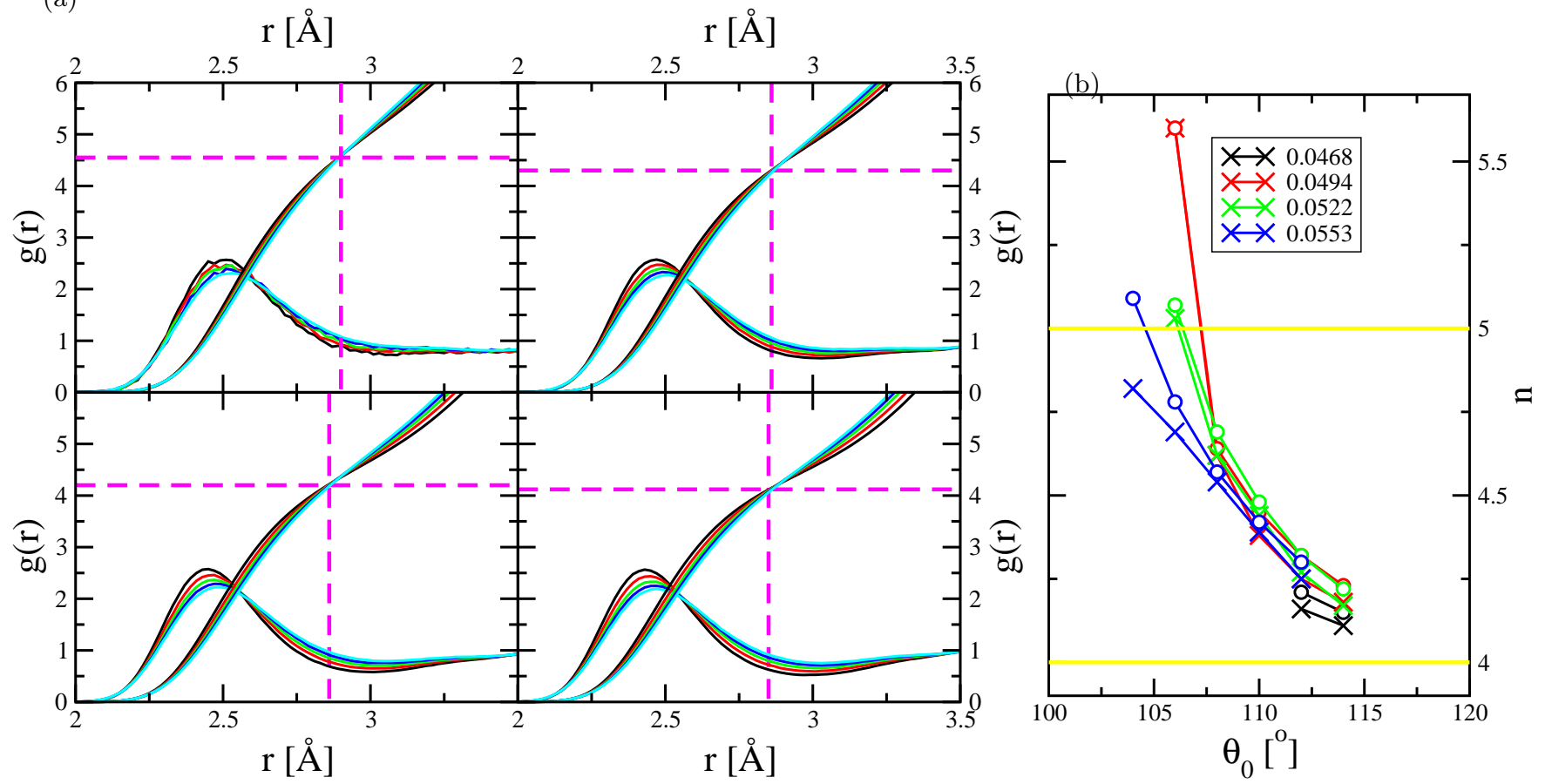

FIG. 3. (a) Radial distribution functions calculated at temperatures around the TMD at fixed density $\left(\rho=0.0494 \AA^{-3}\right)$ for $\theta_{0}=108^{\circ}$ (top left), $110^{\circ}$ (top right), $112^{\circ}$ (bottom left), and $114^{\circ}$ (bottom right) respectively. Functions are shown at temperatures around the TMD temperature of $\Delta T=-200,-100,0,100,200 \mathrm{~K}$ (equivalent to $-0.008,-0.004,0,0.004$, 0.008 in reduced units, respectively) (black, red, green, blue and cyan lines respectively). The figures also show the running coordination numbers, $n=4 \pi \rho \int_{0}^{r} r^{2} g(r) d r$. Each set shows an isobestic point, highlighted with the dashed lines in each panel. (b) Coordination number at the TMD with cutoff corresponding to the isobestic point, shown as a function of both $\rho$ and $\theta_{0}$. The yellow lines highlight the coordination number range of 4-5.

range $4<n_{\text {iso }}<5$ supporting the view that the key to these anomalies is the relationship between the fourth and fifth nearest-neighbours ${ }^{5}$. The divergent behaviour of the TMD lines at high $\lambda$ and $\theta_{0}$ can now be further rationalised. The high temperatures and pressures associated with the TMD lines for these high parameter values support more close-packed structures. However, higher $\theta_{0}$ stabilises lower coordination environments than high $\lambda$ and so the overall coordination number remains closer to four at high $\theta_{0}$ compared with high $\lambda$. The coordination number remains in the critical 4-5 range over a wider $\theta_{0}$ parameter range than for $\lambda$.

To develop a more finely-tuned view of the changes in local coordination environment we evaluate the difference in the mean separations of the fourth and fifth nearest-neighbours, $\Delta r_{45}=r_{5}-r_{4}$, normalised by $r_{5}$. Figures $4(\mathrm{a})$ and (b) show the evolution of $\Delta r_{45}$ as a function of pressure for the TMD lines calculated at different values of $\lambda$ and $\theta_{0}$ respectively. The values of $\Delta r_{45}$ increase as both $\lambda$ and $\theta_{0}$ increase. The figures also show the corresponding ambient pressure $\Delta r_{45}$ values for the key crystal structures (which show stability fields in figure 1). The o-X, BC8 and SC16 crystals show values of $\Delta r_{45}$ consistent with those observed for the TMD lines. The clear implication of figures 3 and 4, therefore, is that the existence of the TMD relies upon a fine balance between open (network-like) structures (here dominated by four-coordinate sites) and close-packed structures (here identified by the move towards the inclusion of the fifth nearest-neighbour into the first coordination shell).

In this work we have demonstrated a correlation between the existence and location of the TMD anomalies in a relatively simple potential model which presents a potentially novel way of rationalising the origin of the anomalous behaviour in liquids. There is a relationship between the TMD lines and the crystal/crystal coexistence curves with the relative distances between the fourth and fifth nearest-neighbour atoms emerging as the key metric. In addition we have reported the limiting values of the potential parameters $\lambda$ and $\theta_{0}$ for which the density anomaly emerges.

\section{ACKNOWLEDGEMENTS.}

DF is grateful to St. Edmund Hall and the Clarendon Fund (University of Oxford) for financial support. We are grateful for support from the EPSRC Centre for Doctoral training, Theory and Modelling in Chemical Sciences, under grant EP/L015722/1. This paper conforms to the RCUK data management requirements. 

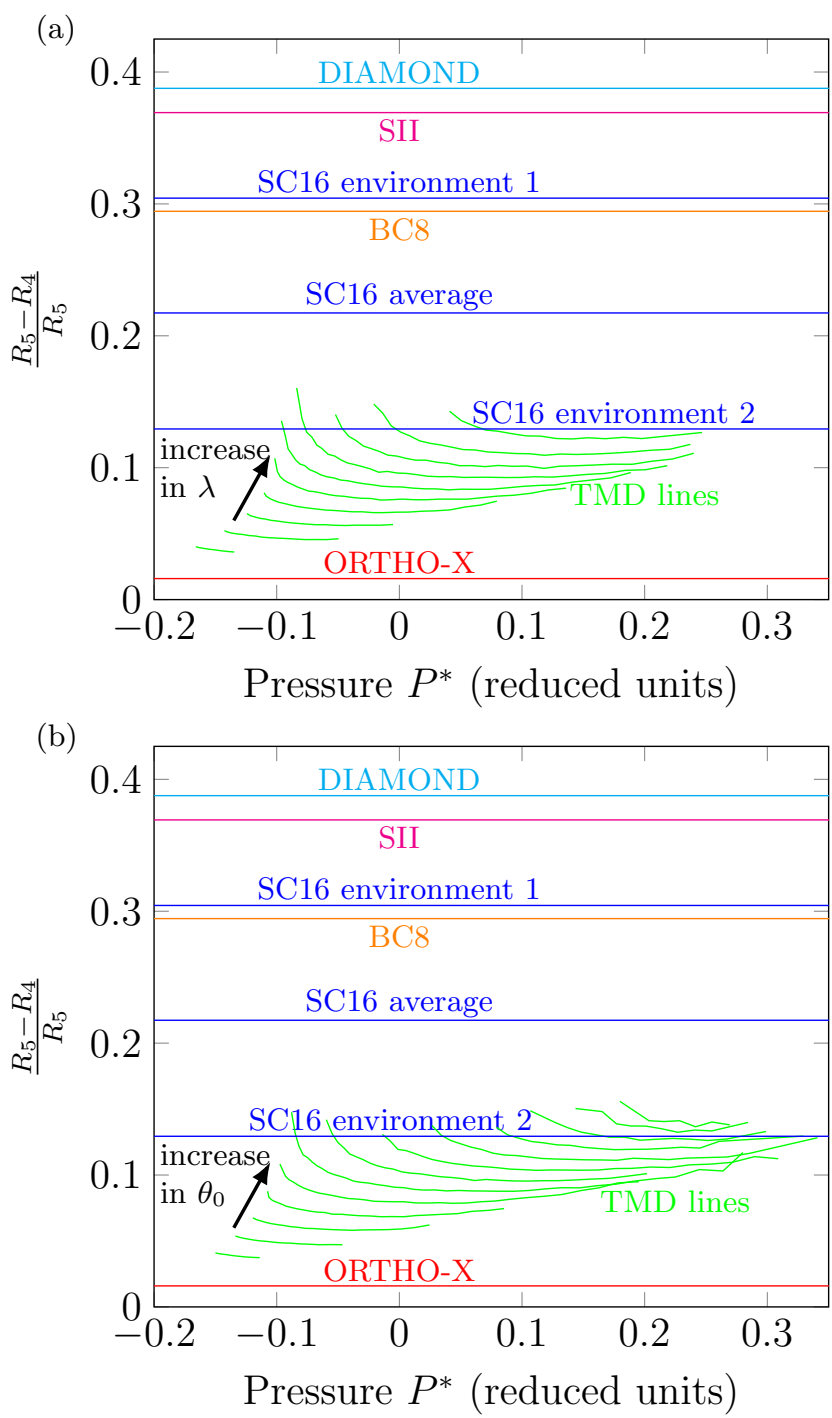

FIG. 4. The distance between the fourth and fifth nearestneighbour atoms, $\Delta r_{45}\left(=\left(r_{5}-r_{4}\right) / r_{5}\right)$, determined at the respective TMDs as a function of reduced pressure, $p^{*}$ and "normalised" by the fifth nearest-neighbour separation, $r_{5}$. The upper and lower panels show $\Delta r_{45}$ as a function of $\theta$ and $\lambda_{0}$ respectively. The respective $(T=0 \mathrm{~K})$ values for the $\mathrm{SC} 16$, $\mathrm{BC} 8$, SII, diamond and o-X crystals, determined at $p^{*}=0$, are shown for comparison. The SC16 crystal shows two such distances and their respective values and the weighted average are shown here.

${ }^{1}$ P. Gallo, K. Amann-Winkel, C. A. Angell, M. A. Anisimov, F. Caupin, C. Chakravarty, E. Lascaris, T. Loerting, A. Z. Panagiotopoulos, J. Russo, J. A. Sellberg, H. E. Stanley, H. Tanaka, C. Vega, L. Xu, and L. G. M. Pettersson, Chemical Reviews 116, 7463 (2016)

${ }^{2}$ R. Shi and H. Tanaka, Proceedings of the National Academy of Sciences , 201717233 (2018)

3 A. Nilsson and L. G. M. Pettersson, Nature Communications 6, 1 (2015)
${ }^{4}$ L. B. Skinner, C. J. Benmore, J. C. Neuefeind, and J. B. Parise, Journal of Chemical Physics 141 (2014), $10.1063 / 1.4902412$

5 J. Russo and H. Tanaka, Nature communications 5, 3556 (2014), arXiv:arXiv:1308.4231v1

6 D. Nayar and C. Chakravarty, Physical Chemistry Chemical Physics 15, 14162 (2013)

7 J. Russo, K. Akahane, and H. Tanaka, Proceedings of the National Academy of Sciences , 201722339 (2018), 
arXiv:arXiv:1408.1149

8 H. Tanaka, Physical Review B - Condensed Matter and Materials Physics 66, 1 (2002)

${ }^{9}$ G. Franzese, G. Malescio, A. Skibinsky, S. V. Buldyrev, and H. E. Stanley, Physical Review E - Statistical, Nonlinear, and Soft Matter Physics 66, 1 (2002), arXiv:0111239 [cond-mat]

10 P. F. McMillan, G. N. Greaves, M. Wilson, M. C. Wilding, and D. Daisenberger, "Polyamorphism and liquidliquid phase transitions in amorphous silicon and supercooled al2o3y2o3 liquids," in Liquid Polymorphism (John Wiley and Sons, Ltd, 2013) pp. 309-353

11 D. Machon, F. Meersman, M. Wilding, M. Wilson, and P. McMillan, Progress in Materials Science 61, 216 (2014)

12 S. Sastry and C. Austen Angell, Nature Materials 2, 739 (2003)

13 P. G. Debenedetti, Journal of Physics Condensed Matter 15, 1669 (2003), arXiv:1708.02002

14 S. Sastry, F. Sciortino, and H. E. Stanley, The Journal of Chemical Physics 98, 9863 (1993)

15 P. H. Poole, F. Sciortino, U. Essmann, and H. E. Stanley, Nature 360, 324 (1992)

16 S. Sastry, P. G. Debenedetti, F. Sciortino, and H. E. Stanley, Phys. Rev. E 53, 6144 (1996)

17 J. R. Errington and P. G. Debenedetti, Nature 409, 318 (2001)

18 M. S. Shell, P. G. Debenedetti, and A. Z. Panagiotopoulos, Physical Review E - Statistical Physics, Plasmas, Fluids, and Related Interdisciplinary Topics 66, 1 (2002), arXiv:0203383 [cond-mat]

19 I. Saika-Voivod, F. Sciortino, and P. H. Poole, Physical Review E 63, 1 (2000), arXiv:0007380 [cond-mat]

20 A. Mudi and C. Chakravarty, Journal of Physical Chemistry B 108, 19607 (2004)

21 A. Mudi and C. Chakravarty, Journal of Physical Chemistry B 110, 4502 (2006)

22 A. Mudi and C. Chakravarty, The Journal of Physical Chemistry B 110, 4502 (2006)

23 A. Mudi, C. Chakravarty, and R. Ramaswamy, The Journal of Chemical Physics 122, 104507 (2005)

24 A. Mudi, C. Chakravarty, and R. Ramaswamy, The Journal of Chemical Physics 124, 069902 (2006)

25 A. Mudi, C. Chakravarty, and E. Milotti, The Journal of Chemical Physics 125, 074508 (2006)

${ }^{26}$ R. Sharma, A. Mudi, and C. Chakravarty, The Journal of Chemical Physics 125, 044705 (2006)

27 R. Sharma, S. N. Chakraborty, and C. Chakravarty, The Journal of Chemical Physics 125, 204501 (2006)

28 M. Agarwal, R. Sharma, and C. Chakravarty, Journal of Chemical Physics 127 (2007), 10.1063/1.2794766

29 M. Agarwal and C. Chakravarty, Journal of Physical Chemistry B 111, 13294 (2007)

30 B. S. Jabes, M. Agarwal, and C. Chakravarty, Journal of Chemical Physics 132 (2010), 10.1063/1.3439593

31 B. Shadrack Jabes, D. Nayar, D. Dhabal, V. Molinero, and C. Chakravarty, Journal of Physics Condensed Matter 24 (2012), 10.1088/0953-8984/24/28/284116

32 G. A. Cisneros, K. T. Wikfeldt, L. Ojamäe, J. Lu, Y. Xu, H. Torabifard, A. P. Bartók, G. Csányi, V. Molinero, and F. Paesani, Chemical Reviews 116, 7501 (2016)
${ }^{33}$ V. Molinero, S. Sastry, and C. A. Angell, Physical Review Letters 97, 1 (2006), arXiv:0510292 [cond-mat]

${ }^{34}$ W. Hujo, B. S. Jabes, V. K. Rana, C. Chakravarty, and V. Molinero, Journal of Statistical Physics 145, 293 (2011), arXiv: 1107.5623

35 M. R. Sadr-Lahijany, A. Scala, S. V. Buldyrev, and H. E. Stanley, Physical Review Letters 81, 4895 (1998)

36 N. B. Wilding and J. E. Magee, Physical Review E 66, 031509 (2002)

37 H. M. Gibson and N. B. Wilding, Physical Review E 73, 061507 (2006)

38 E. Salcedo, A. B. de Oliveira, N. M. Barraz, C. Chakravarty, and M. C. Barbosa, The Journal of Chemical Physics 135, 044517 (2011)

${ }^{39}$ F. H. Stillinger and T. a. Weber, Physical Review B 31, $5262(1985)$

40 J.-W. Jiang, T. Rabczuk, and H. S. Park, Nanoscale 7, $6059(2015)$

41 J.-W. Jiang, Nanotechnology 26, 315706 (2015)

42 M. H. Bhat, V. Molinero, E. Soignard, V. C. Solomon, S. Sastry, J. L. Yarger, and C. A. Angell, Nature 448, 787 (2007)

43 A. S. Barnard and S. P. Russo, Molecular Physics 100, $1517(2002)$

44 V. Molinero and E. B. Moore, The Journal of Physical Chemistry B 113, 4008 (2009)

45 A. Béré and A. Serra, Philosophical Magazine 86, 2159 (2006)

46 Z. Q. Wang, D. Stroud, and A. J. Markworth, Physical Review B 40, 3129 (1989)

47 M. Singh, D. Dhabal, A. H. Nguyen, V. Molinero, and C. Chakravarty, Physical Review Letters 112, 147801 (2014)

48 D. Dhabal, C. Chakravarty, V. Molinero, and H. K. Kashyap, The Journal of Chemical Physics 145, 214502 (2016)

49 C. A. Angell and V. Kapko, Journal of Statistical Mechanics: Theory and Experiment 2016, 094004 (2016)

50 S. Saw, N. L. Ellegaard, W. Kob, and S. Sastry, Physical Review Letters 103, 1 (2009), arXiv:0910.5318

51 N. Kern and D. Frenkel, The Journal of Chemical Physics 118, $9882(2003)$

${ }^{52}$ F. Smallenburg, L. Filion, and F. Sciortino, Nature Physics 10, 653 (2014)

53 J. Crain, S. J. Clark, G. J. Ackland, M. C. Payne, V. Milman, P. D. Hatton, and B. J. Reid, Physical Review B 49, 5329 (1994)

54 J. Crain, R. O. Piltz, G. J. Ackland, S. J. Clark, M. C. Payne, V. Milman, J. S. Lin, P. D. Hatton, and Y. H. Nam, Physical Review B 50, 8389 (1994)

55 K. Akahane, J. Russo, and H. Tanaka, Nature Communications 7, 12599 (2016), arXiv:1611.07148

${ }^{56}$ F. Romano, J. Russo, and H. Tanaka, Phys Rev B Condens Matter 90, 014204 (2014)

57 D. Fijan and M. Wilson, Chemical Physics Letters 685, $316(2017)$

58 P. G. Debenedetti and M. C. D'Antonio, AIChE Journal 34, 447 (1988)

59 L. Bosio, S. H. Chen, and J. Teixeira, Physical Review A 27, 1468 (1983) 